ORIGINAL ARTICLE

\title{
The effect of specialist care within the first year on subsequent outcomes in 24232 adults with new-onset diabetes mellitus: population-based cohort study
}

\author{
F A McAlister, S R Majumdar, D T Eurich, J A Johnson
}

Qual Saf Health Care 2007;16:6-11. doi: 10.1136/qshc.2006.018648

See end of article for authors' affiliations

Correspondence to:

Dr S R Majumdar, 2E307

WMC, University of Alberto Hospital, 8440112 Street, Edmonton, Alberta, Canada T6G 2R7; me2.majumdar@ valberta.ca

Accepted

24 September 2006
Background: Although specialty care has been shown to improve short-term outcomes in patients hospitalised with acute medical conditions, its effect on patients with chronic conditions treated in the ambulatory care setting is less clear.

Objective: To examine whether specialty care (ie, consultative care provided by an endocrinologist or a general internist in concert with a patient's primary care doctor) within the first year of diagnosis is associated with improved outcomes after the first year for adults with diabetes mellitus treated as outpatients.

Design: Population-based cohort study using linked administrative data.

Setting: The province of Saskatchewan, Canada.

Sample: 24232 adults newly diagnosed with diabetes mellitus between 1991 and 2001.

Method: The primary outcome was all-cause mortality. Analyses used multivariate Cox proportional hazards models with time-dependent covariates, propensity scores and case mix variables (demographic, disease severity and comorbidities). In addition, restriction analyses examined the effect of specialist care in low-risk subgroups.

Results: The median age of patients was 61 years, and over a mean follow-up of 4.9 years 2932 (12\%) died. Patients receiving specialty care were younger, had a greater burden of comorbidities, and visited doctors more often before and after their diabetes diagnosis (all $p \leqslant 0.001$ ). Compared with patients seen by primary care doctors alone, patients seen by specialists and primary care doctors were more likely to receive recommended treatments (all $p \leqslant 0.001$ ), but were more likely to die $(13.1 \% v 11.7 \%$, adjusted hazard ratio (HR) $1.17,95 \%$ confidence interval (Cl) 1.08 to 1.27). This association persisted even in patients without comorbidities or target organ damage (adjusted HR 1.16,95\% Cl 1.01 to 1.34).

Conclusion: Specialty care was associated with better disease-specific process measures but not improved survival in adults with diabetes cared for in ambulatory care settings.
A lthough studies often report that specialists are more knowledgeable and more likely to use proved efficacious treatments within their area of expertise than are primary care doctors, the literature asserting improved outcomes with specialist-based care is based largely on short-term outcomes in patients hospitalised with acute myocardial infarction, heart failure, stroke or asthma. ${ }^{1}$ However, for chronic conditions treated largely in the ambulatory care setting, such as diabetes mellitus, the evidence is less definitive.

Although several studies have reported that specialists are more likely to carry out recommended tests $^{2-8}$ or to increase hypoglycaemic treatment, ${ }^{90}$ only one of the seven studies that have examined atherosclerotic risk factor control found significant benefits from specialist care after adjustment for case-mix and selection bias related to referral patterns. ${ }^{2}{ }^{10-14}$ Although one study reported a lower rate of microvascular complications in patients treated by specialists, this difference disappeared after adjustment for differences in case-mix and baseline physiological measures. ${ }^{12}$ All of these studies are limited; they have small numbers, are restricted to highly selected patients, have short durations of follow-up, or focus on disease-specific outcomes (such as glycaemic control). However, focusing on disease-specific outcomes does not consider the possibility that specialist-based care may improve processes and outcomes related to the target condition, but not for other conditions. Of the two studies that investigated allcause mortality in people with diabetes treated by specialists compared with those treated by primary care doctors, one found no difference in 424 patients, ${ }^{13}$ and although the other reported lower mortality in patients attending a diabetes clinic, their analyses were not adjusted for comorbidities. ${ }^{15}$

Thus, we designed this study to examine the relationship between the doctors providing ambulatory care and long-term outcomes in adults with new-onset type 2 diabetes mellitus.

\section{METHODS}

\section{Data sources}

We used the linked databases of Saskatchewan Health (drug, doctors' claim, hospitalisations and vital statistics), which have been described in detail elsewhere, ${ }^{16}$ to examine the relationship between the treating doctor and outcome in patients with diabetes mellitus. These databases are comprehensive and of high quality, have been used in numerous epidemiological studies, ${ }^{16}$ and, unlike similar databases in other jurisdictions, the prescription drug data are not restricted by age.

The Canadian healthcare system is publicly funded and provides universal access to doctor and hospital services. Institutional approval for the study was obtained from the health research ethics board of the University of Alberta.

\section{Patient selection}

Of the 64079 Saskatchewan residents with diabetes (using a definition previously validated for Canadian administrative databases $)^{17}$ who received care between 1991 and 2001, we

Abbreviation: $A C E$, antiotensin-converting enzyme 
excluded those without drug prescription data $(n=4416$ registered Indians, inmates and federal employees), those with diabetes when our study began in 1991 ( $n=20227)$, and those who did not see a doctor again in the first year after diagnosis $(\mathrm{n}=592)$. To limit our sample to patients with type 2 diabetes, we excluded those who were diagnosed with diabetes before 20 years of age $(n=2683)$ and those who were treated exclusively with insulin from the time of diagnosis $(n=637)$. To limit the possibility that our results would be skewed by early clinical events occurring before outpatient care could have any effect, we excluded those patients who were hospitalised within the first year of diagnosis $(n=11292)$.

Thus, our study sample consisted of 24232 adults with a new diagnosis of diabetes after 1991, and who had at least 1 year of continuous coverage under the Saskatchewan health plan.

\section{Exposure to specialty care}

Data on doctors' claims were used to define two groups: those patients who had been seen as an outpatient at least once by a general internist or an endocrinologist in the first year after diagnosis of diabetes were defined as having received "specialty

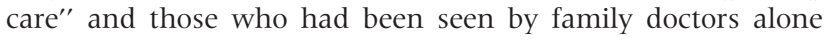
were defined as having received "primary care". It should be noted that over $99 \%$ of the patients who received "specialty care" were still followed up by their primary care doctor on an ongoing basis.

In Canada, as in the UK, patients cannot self-refer themselves to general internists or endocrinologists and neither of these specialist groups provides ongoing primary care. Unlike the USA, general internists act as specialists within the Canadian healthcare system by providing consultative care-indeed, of the 119 medical specialists accepting referrals of patients with diabetes in Saskatchewan, 118 are general internists (College of Physicians and Surgeons of Saskatchewan physician registry, searched 8 Aug 2006). Although primary care doctors in Canada can practice independently after only $1-2$ years of postgraduate training, both general internists and endocrinologists have to complete a minimum of 4 years of hospital-based postgraduate training (the first 3 years of which are common between these specialty streams) and pass written and oral examinations in internal medicine of the Royal College of Physicians and Surgeons of Canada.

\section{Outcomes}

Our primary outcome was all-cause mortality, and secondary outcomes included new prescriptions for drugs specific for diabetes and new prescriptions for cardiovascular drugs recommended for adult patients with diabetes (statins, antiplatelet agents and angiotensin-converting enzyme (ACE) inhibitors). ${ }^{18}$ All patients were prospectively followed up until death, emigration from Saskatchewan, or 31 December 2001, whichever came first.

\section{Analysis}

We used $\chi^{2}$ and Kruskal-Wallis tests to compare baseline characteristics across groups, Kaplan-Meier analysis to examine unadjusted survival patterns across study groups, and Cox proportional hazards regression models to assess the independent relationship between the type of treating doctor and outcomes. Potential confounding variables included as fixed covariates in all multivariate models were age, sex, rural/urban residence, total visits to doctor before diagnosis of diabetes, comorbidities at baseline and the modified Chronic Disease Score. ${ }^{19-22}$ The Chronic Disease Score provides an indication of the burden of comorbidity by identifying specific drug treatments prescribed during the follow-up period, and has been shown to predict resource use, hospitalisation and mortality. ${ }^{19-23}$
To adjust for potential selection bias (confounding by indication), we calculated a propensity score for specialist care using standard methods (logistic regression modelling exposure to specialist against potential confounding variables, but without considering the outcomes) and included this as a fixed covariate in all multivariate models. ${ }^{24}$ Finally, to control for survivor bias, we used time-dependent variables to adjust for the cumulative number of visits to a doctor after diagnosis of diabetes. Proportionality assumptions for the Cox model were tested with both the goodness-of-fit and log-minus-log tests and met for all analyses. All first-order interaction terms were tested in these models; as none had $\mathrm{p}<0.20$, none were included in our final models.

\section{Sensitivity analyses}

By using a previously published approach to minimise the effect of confounding by unmeasured comorbidities, ${ }^{25}$ we examined the effect of specialty care in the healthiest subgroups (patients without documented comorbidities or atherosclerotic target organ damage)—we hypothesised that any differences in outcomes between different ambulatory care physicians should be least subject to confounding in this group.

In three sensitivity analyses we redefined "specialty care" on the basis of: (1) threshold (with only those who had at least three outpatient visits with a specialist in the first year after diagnosis of diabetes being classified as receiving specialty care); (2) dosage (defined as the percentage of total outpatient visits that were with a specialist, and grouped into $<25 \%, 25-$ $50 \%$ and $>50 \%$ ); and (3) timing (with only those referred within the first 6 months after diagnosis compared with those who were referred later in the first year).

\section{RESULTS}

The median age at diagnosis of diabetes was 61 years. Patients referred to a specialist by their primary care doctor in the first year after diagnosis of diabetes were younger, more likely to be men, more likely to be from an urban setting, had a greater burden of comorbidities and visited doctors more often than patients seen solely by primary care doctors (table 1).

Of patients receiving specialty care, 2134 (31\%) had $\geqslant 3$ specialist visits within the first year and specialist visits accounted for more than half of the total outpatient visits in that first year in $706(10 \%)$ patients. Only $114(0.5 \%)$ patients were treated solely by a specialist after diagnosis of diabetesall other patients in the specialty care group were also seen on an ongoing basis by their primary care doctor (indeed, specialty care patients averaged almost two extra visits to their primary care physician in the first year after diagnosis; table 1).

\section{Use of diabetes-specific treatments}

Over the mean follow-up of 4.9 years in this study, patients receiving specialty care were more likely to be started on insulin $(996 / 6842(15 \%) \vee 1672 / 17390(10 \%), \mathrm{p}<0.001)$ and were more likely to be prescribed $\geqslant 2$ oral hypoglycaemic agents (1935/ $6842(28 \%) v 4488 / 17390(26 \%), \mathrm{p}<0.001)$ than primary care patients. The association between increased insulin use and specialty care was maintained in all adjusted analyses (fig 1). Furthermore, specialty care patients who were started on insulin were started significantly sooner than primary care patients (mean $4.5 \vee 4.7$ years, $\mathrm{p}<0.001$ ).

\section{Use of cardiovascular drugs}

Specialty care patients were more likely than primary care patients to be started on cardiovascular agents recommended for adult patients with diabetes $(\mathrm{p}<0.001): 21 \% v 15 \%$ for statins, $19 \%$ v $14 \%$ for antiplatelet agents, $41 \% v 38 \%$ for ACE inhibitors and $5 \% \vee 3 \%$ for all three agents. The association between 


\begin{tabular}{|c|c|c|c|}
\hline Characteristic & $\begin{array}{l}\text { Primary care } \\
(n=17390)\end{array}$ & $\begin{array}{l}\text { Specialty care } \\
(n=6842)\end{array}$ & p Value \\
\hline Age, years (mean (SD)) & $61(15)$ & $59.5(14.7)$ & $<0.001$ \\
\hline Men, $\mathrm{n}(\%)$ & $9582(55)$ & $3926(57)$ & 0.001 \\
\hline Practice setting, $n(\%)$ & & & $<0.001$ \\
\hline Large urban & 5609 (32) & $3150(46)$ & \\
\hline Small urban & $3414(20)$ & $1147(17)$ & \\
\hline Rural & $8367(48)$ & $2545(37)$ & \\
\hline $\begin{array}{l}\text { Total visits to a doctor in the year before } \\
\text { diagnosis of diabetes (mean (SD)) }\end{array}$ & $7.8(8.6)$ & $10.5(10.8)$ & $<0.001$ \\
\hline \multicolumn{4}{|l|}{ Comorbidities at baseline, $\mathrm{n}(\%)$} \\
\hline Coronary artery disease & $1898(11)$ & $1577(23)$ & $<0.001$ \\
\hline Stroke & $357(2)$ & $249(4)$ & $<0.001$ \\
\hline Diabetic neuropathy & $200(1)$ & $152(2)$ & $<0.001$ \\
\hline Diabetic retinopathy & $1430(8)$ & $645(9)$ & 0.003 \\
\hline Diabetes nephropathy & $525(3)$ & 267 (4) & $<0.001$ \\
\hline \multicolumn{4}{|c|}{ Resource use within the first year of diabetes diagnosis, (mean (SD)) } \\
\hline Primary care visits within the 1 year & $9.6(7.5)$ & $11.5(9)$ & $<0.001$ \\
\hline Specialist visits within the 1 year & 0 & $2.5(3.0)$ & $<0.001$ \\
\hline
\end{tabular}

cardiovascular drug use and specialty care was maintained even after adjustment for all covariates including time-dependent covariates and propensity scores (adjusted hazard ratio (HR) $1.19,95 \%$ confidence interval (CI) 1.01 to 1.40 ).

Subgroup analyses confirmed that patients with coronary artery disease at baseline were more likely to be prescribed statins $(28 \% \vee 17 \%, \mathrm{p}<0.001)$ and those with heart failure at baseline were more likely to be prescribed an ACE inhibitor $(41 \% \vee 38 \%, \mathrm{p}<0.001)$ if they received specialty care.

\section{All-cause mortality}

Over 5 years of follow-up, all-cause mortality was higher in specialty care patients $(893 / 6842,13.1 \%)$ than patients cared for solely by primary care doctors (2039/17 390, $11.7 \%$; fig 2$)$. The unadjusted mortality risk in specialty care patients was 1.15 (95\% CI 1.07 to 1.25 ) compared with primary care patients; the mortality excess in specialty care patients remained significant in models adjusting for case mix, propensity score and time-varying covariates (adjusted HR 1.17, 95\% CI 1.08 to 1.27, fig 3). In addition, this association persisted in all low-risk subgroups (fig 4) regardless of whether the definition of specialty care was restricted to only those patients who saw a specialist at least three times per year (adjusted HR 1.34, 95\% CI 1.18 to 1.52 ) or only those who saw a specialist within the first 6 months of diagnosis (adjusted HR 1.32, 95\% CI 1.19 to 1.47). There was also a doseresponse gradient; compared with those patients who had all of their outpatient visits with a primary care doctor, the adjusted HR was 1.09 (95\% CI 0.996 to 1.20 ) for those receiving $<25 \%$ of their outpatient care from a specialist, 1.14 (95\% CI 0.97 to 1.35 ) for those receiving $25-50 \%$ of their outpatient care from a specialist, and 2.04 (95\% CI 1.69 to 2.45 ) for those who had $>50 \%$ of their outpatient visits with a specialist.

\section{DISCUSSION}

In our study of adults newly diagnosed with diabetes in a publicly funded healthcare system with universal access, those

\section{Insulin use}

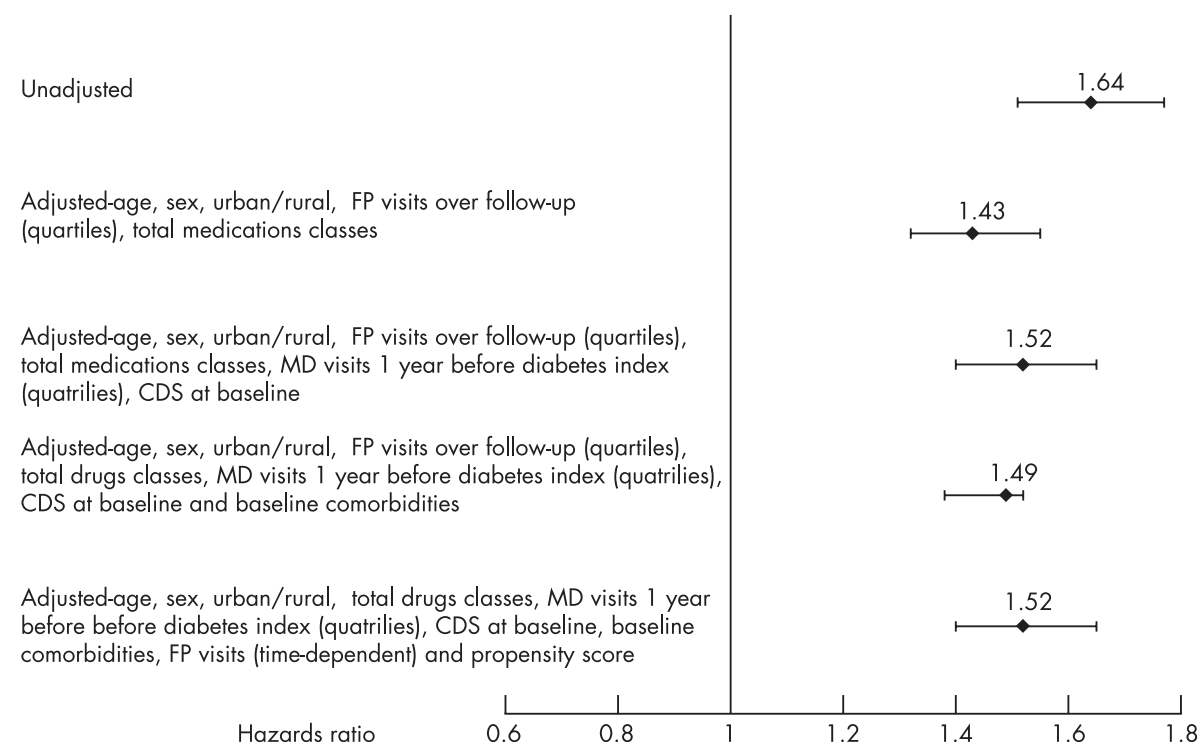

Figure 1 Use of insulin. CDS, Chronic Disease Score (an indication of burden of comorbidity based on number and type of drugs prescribed during the follow-up period); FP, family physician. 


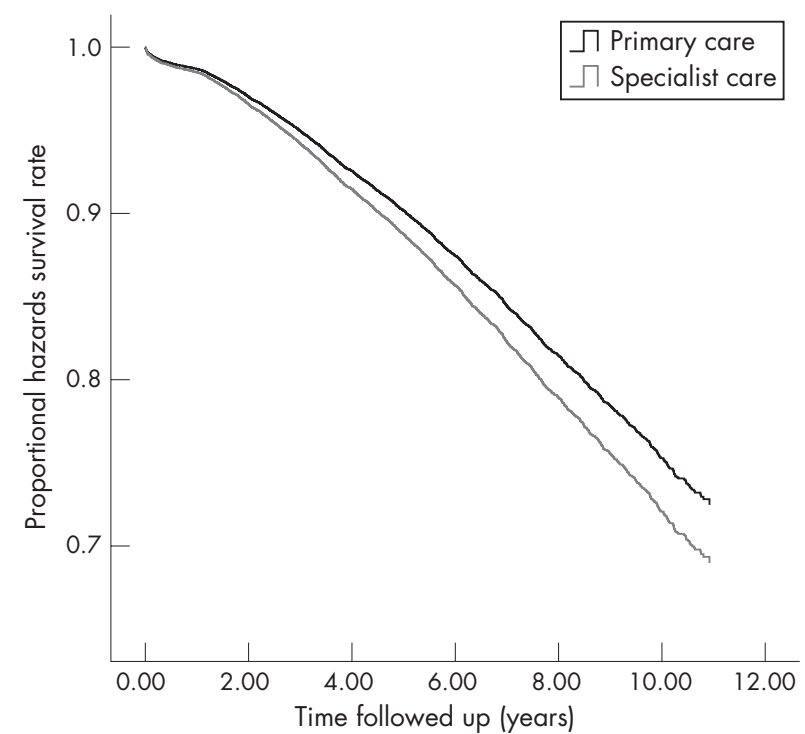

Figure 2 Kaplan-Meier graph of survival in patients treated by specialists or primary care doctors (crude mortality).

referred for specialist assessment were younger, more likely to be men, more often from large urban centres, and had a higher burden of comorbidities than patients cared for solely by primary care doctors. Patients receiving specialty care were seen more often (both by their primary care physician and by all doctors), were more likely to be treated with insulin and combination oral hypoglycaemic agents, and more likely to receive treatment proved efficacious in preventing atherosclerotic complications. Despite these advantages, patients receiving specialty care did not exhibit improved survival, even after adjustment for covariates and even in subgroups without comorbidities or target organ damage at baseline.

Although our findings may merely reflect confounding by indication (ie, specialists are only referred sicker patients with more comorbidities or more severe disease who are more likely to die), we adjusted for all available prognostic factors, restricted our analyses to incident cases of diabetes, dealt with time-varying exposures that could lead to survivor bias, used propensity scores to minimise selection bias related to specialist referral, performed sensitivity analyses to examine different definitions for specialty care, and attempted to minimise confounding by performing fully adjusted analyses in low-risk patient subgroups without comorbidities or target organ damage. Importantly, the magnitude of the association between specialty care and mortality was not appreciably reduced with any of these adjustments (and in some patients was even increased), and the association was consistent in magnitude and directionality across all examined subgroups. An unmeasured confounder would have had to be three times more common among patients treated in primary care and associated with an approximate 3\% absolute mortality benefit to appreciably alter our results.

Given the absence of data supporting the converse viewpoint that specialty care for patients with diabetes improves the mortality, perhaps the assumption that specialty care is better for all patients, all conditions, and in all circumstances deserves re-examination. Several recent studies have documented that although healthcare expenditures are greater in regions (or healthcare delivery systems) with higher-intensity specialistdriven practice patterns compared with regions or systems exhibiting more conservative primary-care-based practice patterns, quality of care and outcomes are not appreciably better (and, for some preventive services such as influenza vaccination and cancer screening, in fact worse). ${ }^{26-30}$ To paraphrase Elliott Fisher, "more is not always better". ${ }^{31} 32$ Is it plausible that specialty care for people with type 2 diabetes might be associated with poorer outcomes? Certainly, the fragmentation of care that can occur when more than one doctor becomes involved with a patient may negatively affect the outcomes (for instance, one can imagine situations in which both doctors may assume that the other is dealing with non-diabetes-related issues such as flu vaccinations or cancer screening)..$^{33}$ Indeed, a study on elderly Canadians showed that patients who saw more than one doctor were at increased risk for receiving an inappropriate drug combination. ${ }^{34}$ In our study, we did find a dose-response gradient in the association between specialty care and mortality; within the specialty care group, those

All-cause mortality

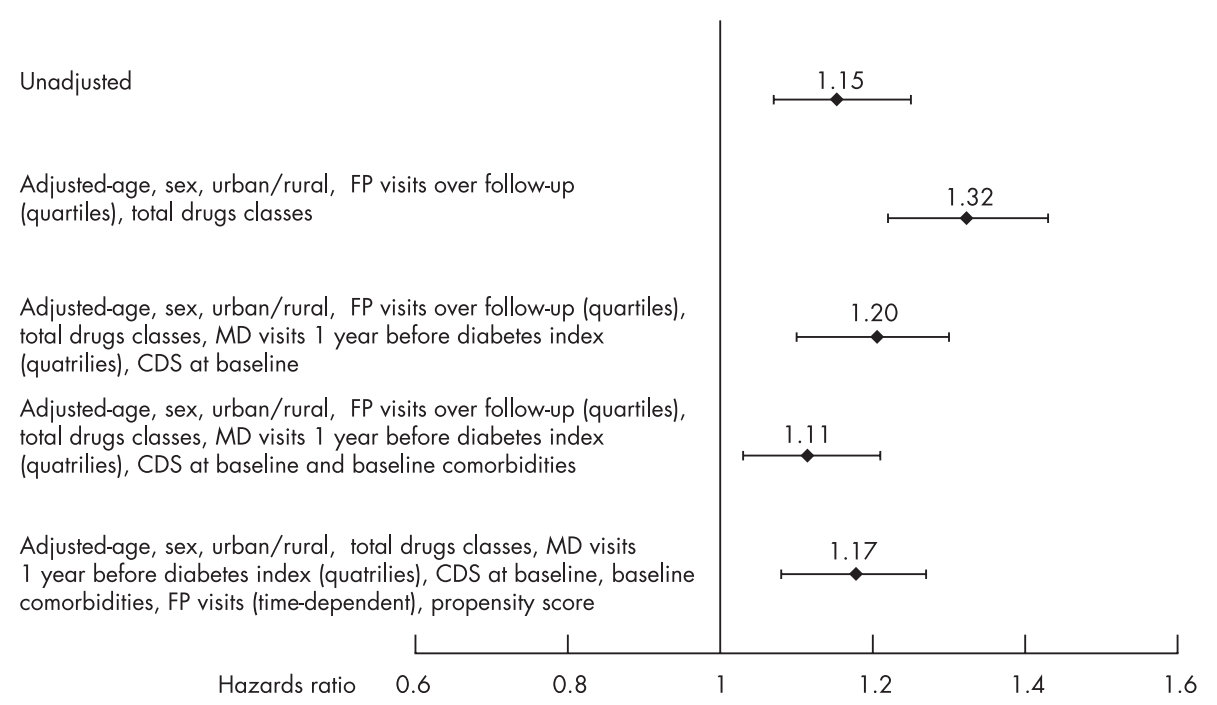

Figure 3 All-cause mortality $>5$ years, crude and adjusted. CDS, Chronic Disease Score (an indication of burden of comorbidity based on number and type of drugs prescribed during the follow-up period); FP, family physician. 


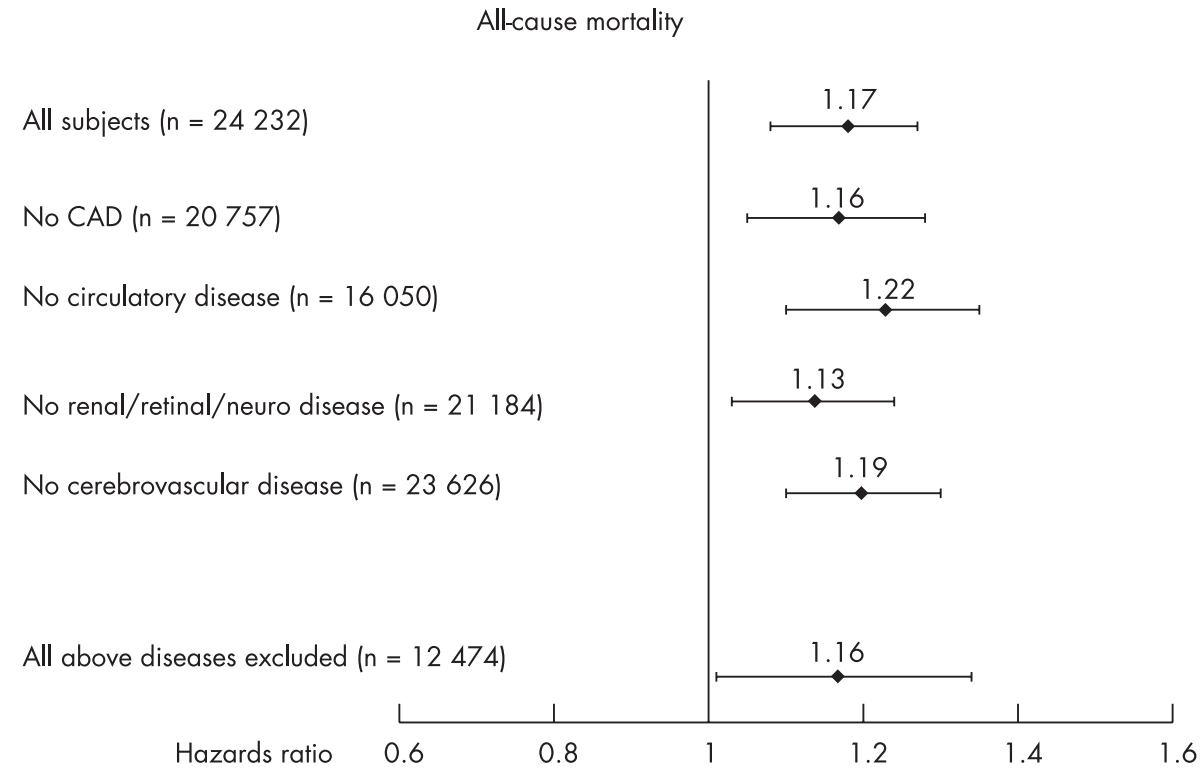

Figure 4 Adjusted analyses for 5-year all-cause mortality in low-risk subgroups. CAD, coronary artery disease; renal/retinal/neuro disease, diabetic microvascular retinopathy/nephropathy/neuropathy.

patients who received a greater percentage of their ambulatory care from a specialist had poorer outcomes than those who received most of their outpatient care from a primary care doctor. Secondly, although specialists are more likely to order tests and treatments for their target condition, they are also less likely to investigate or treat other conditions outside their area of specialty. ${ }^{7536}$ Thus, for a condition such as diabetes, in which 5-year mortality is driven more by cardiovascular than diabetes-related events, the benefits of a disease-specific specialist may be less than for other conditions (such as heart failure) in which the target condition accounts for the bulk of subsequent mortality within a relatively short timeframe. ${ }^{37}$ Finally, perhaps doctors' specialty is less important than their experience with the target condition; for a common condition such as diabetes, experienced primary care doctors will have dealt with more patients than junior specialists.

\section{Limitations}

Although our study includes a large, representative and population-based sample of all adults with newly diagnosed diabetes and all doctors in Saskatchewan, followed up for a long period of time, and our analyses were adjusted for covariates known to affect outcomes in patients with diabetes, there are some limitations to our study. Firstly, we did not have data on physiological parameters such as blood pressure, cholesterol levels or glycaemic control in our study subjects; thus, we cannot say whether specialty care patients had poorer outcomes because their metabolic parameters were less optimal. In a related vein, we do not have data on severity of comorbidities and thus can only adjust for the presence or absence of the comorbidities listed in table 1 in our analyses (although we did include in our adjustments the Chronic Disease Score, which has been shown to perform well as a proxy for disease severity). ${ }^{20-23}$ Secondly, we focused on all-cause mortality, and there may well be nonmortality benefits arising from specialty care that our study was unable to capture. Thirdly, as over $99 \%$ of our patients who received specialty care continued to be followed up by their primary care doctor, our study does not provide information on the effect of pure specialty care in patients with diabetes (eg, a system whereby all patients with diabetes are referred to, and subsequently followed up indefinitely by, a specialised diabetes service without involvement of their primary care doctor). Although such a system has been shown to be efficacious for patients with heart failure deemed to be at high risk for hospitalisation,,$^{38}$ the practicality of such a system for diabetes (which is far more prevalent and associated with a much greater survival time) remains to be investigated. Thus, our study highlights the need for ongoing evaluation of different models of care provision, and in particular the need for high-quality evidence evaluating the effect of specialty care for chronic conditions dealt with in ambulatory care settings (such as diabetes mellitus) with attention to health-related quality of life as well as measures of health resource use, morbidity and mortality.

In conclusion, despite the common assumption that specialty care for patients with chronic conditions invariably improves the quality of their care and their prognosis, our study suggests that specialty care can improve disease-specific process measures without necessarily improving overall health outcomes. Although we showed a robust association between receiving specialty care and poorer outcomes, which persisted through all adjustments and sensitivity analyses, our study is merely observational and we are certainly not suggesting that specialists cause adverse outcomes. However, we believe that our study should raise the awareness that specialty care may not necessarily improve the clinical outcomes for all conditions.

\section{ACKNOWLEDGEMENTS}

We thank Dr Richard Lewanczuk for his comments on our manuscript. This study is based on non-identifiable data provided by the Saskatchewan Department of Health. The interpretation and conclusions contained herein do not necessarily represent those of the Government of Saskatchewan or the Saskatchewan Department of Health

\section{Authors' affiliations \\ F A McAlister, S R Majumdar, D T Eurich, J A Johnson, Department of Medicine, University of Alberta, Edmonton, Alberta, Canada}

Funding: DTE holds a full-time studentship in health research from the Alberta Heritage Foundation for Medical Research (AHFMR). SRM and FAM receive salary from the Canadian Institutes of Health Research (CIHR) and the AHFMR. FAM holds the University of Alberta/Merck Frosst/Aventis 
Chair in patient health management. JAJ is a health scholar with the AHFMR and holds a Canada Research Chair in diabetes health outcomes. JAJ is the chairman of a New Emerging Team (NET) grant to the Alliance for Canadian Health Outcomes Research in Diabetes (ACHORD). The ACHORD NET grant is sponsored by the Canadian Diabetes Association, the Heart and Stroke Foundation of Canada, The Kidney Foundation of Canada, the ClHR - Institute of Nutrition, Metabolism and Diabetes, and the ClHR - Institute of Circulatory and Respiratory Health. None of these sponsoring agencies had any input into the design, conduct or reporting of this study.

Competing interests: None.

\section{REFERENCES}

1 Harrold LR, Field TS, Gurwitz JH. Knowledge, patterns of care, and outcomes of care for generalists and specialists. J Gen Intern Med 1999;14:499-51 1.

2 De Berardis G, Pellegrini F, Franciosi M, et al. Quality of care and outcomes in type 2 diabetic patients: a comparison between general practice and diabetes clinics. Diabetes Care 2004:27:398-406.

3 Greenfield S, Kaplan SH, Kahn R, et al. Profiling care provided by different groups of physicians: effects of patient case-mix (bias) and physician-level clustering on quality assessment results. Ann Intern Med 2002;136:111-21.

4 Zgibor JC, Songer Ti, Kelsey SF, et al. The association of diabetes specialist care with health care practices and glycemic control in patients with type 1 diabetes: a cross-sectional analysis from the Pittsburgh Epidemiology of Diabetes Complications Study. Diabetes Care 2000;23:472-6.

5 Ho $M$, Marger M, Beart J, et al. Is the quality of diabetes care better in a diabetes clinic or in a general medicine clinic? Diabetes Care 1997;20:472-5.

6 Chin MH, Zhang JX, Merrell K. Specialty differences in the care of older patients with diabetes. Med Care 2000;38:131-40.

7 Lafata JE, Martin S, Morlock R, et al. Provider type and the receipt of general and diabetes-related preventive health services among patients with diabetes. Med Care 2001;39:491-9.

8 Weiner JP, Parente ST, Garnick DW, et al. Variation in office-based quality. A claims-based profile of care provided to Medicare patients with diabetes. JAMA 1995:273:1503-8.

9 Shah BR, Hux JE, Laupacis A, et al. Clinical inertia in response to inadequate glycemic control. Do specialists differ from primary care physicians? Diabetes Care 2005;28:600-6.

10 Chin MH, Su AW, Jin L, et al. Variations in the care of elderly persons with diabetes among endocrinologists, general internists, and geriatricians. J Gerontol Med Sci 2000;55A:M601-6

11 Shah BR, Hux JE, Laupacis A, et al. Diabetic patients with prior specialist care have better glycaemic control than those with prior primary care. J Eval Clin Pract 2005;11:568-75.

12 Zgibor JC, Songer TJ, Kelsey SF, et al. Influence of health care providers on the development of diabetes complications. Long term follow-up from the Pittsburgh Epidemiology of Diabetes Complications Study. Diabetes Care 2002:25:1584-90.

13 Greenfield S, Rogers W, Mangotich M, et al. Outcomes of patients with hypertension and non-insulin-dependent diabetes mellitus treated by different systems and specialties. Results from the Medical Outcomes Study. JAMA 1995:274:1436-44.

14 Ismail H, Wright J, Rhodes P, et al. Quality of care in diabetic patients attending routine primary care clinics compared with those attending GP specialist clinics. Diabet Med 2006;23:857-66.
15 Verlato G, Muggeo M, Bonora E, et al. Attending the diabetes center is associated with increased 5-year survival probability of diabetic patients. The Verona Diabetes Study. Diabetes Care 1996;19:211-13.

16 Downey W, Beck P, McNutt M, et al. Health databases in Saskatchewan, 3rd edn. In: Strom BL, ed. Pharmacoepidemiology. Chichester: Wiley, 2000:325-45

17 Hux JE, Ivis F, Flintoft V, et al. Diabetes in Ontario: determination of prevalence and incidence using a validated administrative data algorithm. Diabetes Care 2002;25:512-16.

18 Brown LC, Johnson JA, Majumdar SR, et al. The management of cardiovascular risk in patients with type 2 diabetes mellitus and symptomatic atherosclerosis is suboptimal. CMAJ 2004;171:1189-92.

19 Johnson JA, Majumdar SR, Simpson SH, et al. Decreased mortality associated with metformin use compared to sulfonylurea monotherapy in type 2 diabetes mellitus. Diabetes Care 2002;25:2244-8.

20 Clark DO, Von Korff M, Saunders K, et al. A chronic disease score with empirically derived weights. Med Care 1995;33:783-95.

21 Johnson RE, Hornbrook MC, Nichols GA. Replicating the chronic disease score (CDS) from automated pharmacy data. J Clin Epidemiol 1994:47:1191-9.

22 Von Korff M, Wagner EH, Saunders K. A chronic disease score from automated pharmacy data. J Clin Epidemiol 1992;45:197-203.

23 Schneeweiss S, Maclure M. Use of comorbidity scores for control of confounding in studies using administrative databases. Int J Epidemiol 2000;29:891-8.

24 Rubin DB. Estimating causal effects from large data sets using propensity scores. Ann Intern Med 1997; 127:757-63.

25 Wen SW, Hernandez R, Naylor CD. Piffalls in nonrandomized outcome studies: the case of incidental appendectomy with open cholecystectomy. JAMA 1995;274:1687-91.

26 Guadagnoli E, Hauptman PJ, Ayanian JZ, et al. Variation in the use of cardiac procedures after acute myocardial infarction. $N$ Engl J Med 1995;333:573-8.

27 Jha AK, Perlin JB, Kizer KW, et al. Effect of the transformation of the Veterans Affairs health care system on the quality of care. N Engl J Med 2003:348:2218-27.

28 Fisher ES, Wennberg DE, Stukel TA, et al. The implications of regional variations in Medicare spending. Part 1: the content, quality, and accessibility of care. Ann Intern Med 2003;138:273-87.

29 Fisher ES, Wennberg DE, Stukel TA, et al. The implications of regional variations in Medicare spending. Part 2: health outcomes and satisfaction with care. Ann Intern Med 2003; 138:288-98.

30 Phelps CE. What's enough, what's too much? Ann Intern Med 2003;138:348-9.

31 Fisher ES. Medical care-Is more always better? N Engl J Med 2003:349:1665-7.

32 Fisher ES, Welch HG. Avoiding the unintended consequences of growth in medical care. How might more be worse? JAMA 1999;281:446-53.

33 van Walraven C, Mamdani M, Fang J, et al. Continuity of care and patient outcomes after hospital discharge. J Gen Intern Med 2004;19:624-31.

34 Tamblyn RM, McLeod PJ, Abrahamowicz M, et al. Do too many cooks spoil the broth? Multiple physician involvement in medical management of elderly patients and potentially inappropriate drug combinations. CMAJ 1996;154:1 177-84.

35 Redelmeier DA, Tan SH, Booth GL. The treatment of unrelated disorders in patients with chronic medical diseases. N Engl J Med 1998:338:1516-20.

36 Fontana SA, Baumann LC, Helberg C, et al. The delivery of preventive services in primary care practices according to Chronic Disease Status. Am J Public Health 1997;87:1190-6.

37 Ezekowitz J, van Walraven C, McAlister FA, et al. Impact of specialist follow-up in outpatients with congestive heart failure. CMAJ 2005;172:189-94.

38 McAlister FA, Stewart S, Ferrua S, et al. Multidisciplinary strategies for the management of heart failure patients at high risk for admission: a systematic review of randomized trials. J Am Coll Cardiol 2004;44:810-19. 